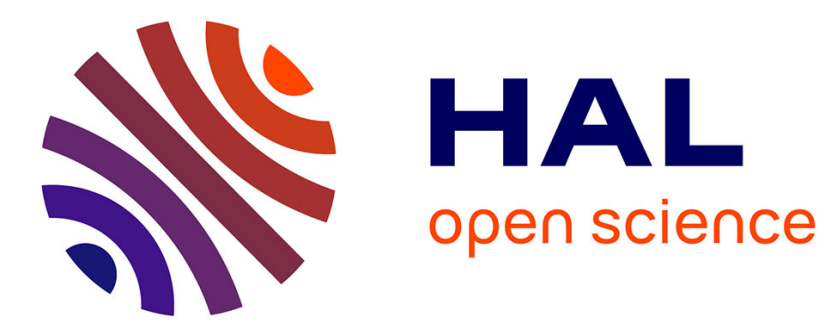

\title{
Macroscopic model of fluid structure interaction in cylinder arrangement using theory of mixture
}

\author{
A Gineau, E. Longatte, D. Lucor, P. Sagaut
}

\section{To cite this version:}

A Gineau, E. Longatte, D. Lucor, P. Sagaut. Macroscopic model of fluid structure interaction in cylinder arrangement using theory of mixture. Computers and Fluids, 2020, 202, pp.104499. 10.1016/j.compfluid.2020.104499 . hal-03251640

\section{HAL Id: hal-03251640 \\ https://hal.science/hal-03251640}

Submitted on 7 Jun 2021

HAL is a multi-disciplinary open access archive for the deposit and dissemination of scientific research documents, whether they are published or not. The documents may come from teaching and research institutions in France or abroad, or from public or private research centers.
L'archive ouverte pluridisciplinaire HAL, est destinée au dépôt et à la diffusion de documents scientifiques de niveau recherche, publiés ou non, émanant des établissements d'enseignement et de recherche français ou étrangers, des laboratoires publics ou privés. 


\title{
Macroscopic model of fluid structure interaction in cylinder arrangement using theory of mixture
}

\author{
A. Gineau ${ }^{\mathrm{a}, \mathrm{b}}$, E. Longatte La,* D. Lucor $^{\mathrm{b}}$, P. Sagaut ${ }^{\mathrm{b}}$ \\ a Laboratoire de Mécanique des Structures Industrielles Durables, UMR EDF/CNRS/CEA 8193, Clamart, France \\ b Sorbonne Universités, UPMC P6, Institut Jean le Rond d'Alembert, UMR 7190, Paris, France
}

\section{A R T I C L E I N F O}

Article history:

Received 10 July 2018

Revised 30 October 2019

Accepted 6 March 2020

Available online 7 March 2020

\begin{abstract}
A B S T R A C T
In the framework of the theory of mixture, the dynamic behaviour of solid cylinder bundles submitted to external hydrodynamic load exerted by surrounding viscous fluid flow is described. Mass conservation and momentum balance formulated on an elementary domain made of a given volume of mixture give rise to a system of coupled equations governing solid space-averaged displacement, fluid velocity and pressure provided that near-wall hydrodynamic load on each vibrating cylinder is expressed as a function of both fluid and solid space-averaged velocity fields.

Then, the ability of the macroscopic model to reproduce over time an averaged flow surrounding vibrating cylinders in a large array in the context of small magnitude displacements is pointed out. Numerical solutions obtained on a two-dimensional configuration involving an array of several hundreds of cylinders subjected to an impulsional load are compared to those provided by averaged well-resolved microscopic-scale solutions. The relative error is less than $3 \%$ in terms of displacement magnitude and $5 \%$ for frequency delay.

The proposed macroscopic model does not include any assumption on relative effect contributions to mechanical exchanges occurring in the full domain. Therefore it features interesting properties in terms of fluid solid interaction prediction capabilities. Moreover it contributes to a significant gain in terms of computational time and resources. Further developments are now required in order to extent the formulation to large magnitude displacements including three-dimensional effects. This could be recommended for investigations on fuel assembly vibration risk assessment in Pressure Water, Fast Breeder reactors at a whole core scale or any other large-scale mechanical system involving some kind of periodic geometry.
\end{abstract}

\section{Introduction}

Modeling mechanical interaction between fluid and solid in a large array of vibrating slender-bodies is a matter of design and safety assessment in nuclear installations. A detailed microscopicscale representation of interstitial flow patterns may be computationally unreachable for size purposes. Therefore an alternative consists in describing each cylinder dynamic behaviour from a global point of view by "forgetting" phenomena occurring below the scale of interest $[3,6,20,24]$. In the present work, one considers a multi-phase fluid-solid system made of a periodic arrangement of cylinders submitted to a single-phase viscous unsteady fluid flow inducing external load and vibration. A specific attention

\footnotetext{
* Corresponding author.

E-mail address: elongatte@gmail.com (E. Longatte).
}

is paid to the description of each cylinder motion and associated interstitial fluid dynamics.

According to the theory of mixture, the purpose is to formulate a macroscopic model for both fluid and solid phase dynamics at a larger scale than the microscopic scale. In the present article it is developed in a two-dimensional context $[4,9]$. One considers an elementary volume $\Omega$ of mixture defined such that it contains only one cylinder (Fig. 1). Each cylinder displacement magnitude is strictly limited within the boundary of $\Omega$ and the model is built such that the hydrodynamic load arising at fluid solid interface is related to macroscopic scale quantities, namely the averaged flow fields and the averaged solid response over $\Omega$.

Within $\Omega$ the solid phase domain is denoted by $\Omega_{s}$ (of volume $V_{s}$ ) of boundary $\partial \Omega_{S}$ (of area $S_{S}$ ) and the fluid phase domain by $\Omega_{f}$ (of volume $V_{f}$ ) of boundary $\partial \Omega_{f}$ (of area $S_{f}$ ). Therefore, one gets $\Omega=\Omega_{s} \cup \Omega_{f}$ as displayed on Fig. 1. At microscopic scale space and time evolution of any point belonging to $\Omega$ may be described by local mass density $\rho(\mathbf{x}, t)$ and velocity $\mathbf{v}(\mathbf{x}, t)$. In the framework of 


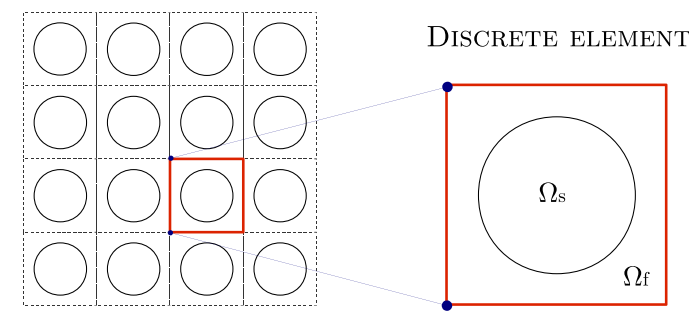

Fig. 1. One discrete element within a square arrangement of cylinders.

theory of mixture, assumptions are required to formulate balance laws on the volume of fluid solid mixture. $\alpha$-phase represents either solid or fluid phase such that $\alpha \in\{s ; f\}$.

Theory of mixture is used to describe mechanical behaviour and transport in multi-phase system such as porous media or flow of immiscible fluid. Such a system consists of several continuous media interlinked to each other by sharp interfaces. Each phase is characterized by its usual material patterns so that in the framework of classical continuum media theory, a solution for its mechanical behaviour, together with interfacial conditions, can be obtained at a space scale referred to as the microscopic scale $[7,8,22,23]$. If information at microscopic scale is not required, multi-phase solution at such a small space scale is not necessary. In this way theory of mixture provides an averaged description of the multi-phase system within a volume whose size is much larger than the microscopic scale referred to as the macroscopic scale. This yields to macroscopic governing equations for system to be considered over a given volume where balance laws have to be satisfied. This approach represents an extension of classical balance laws in the context of continuum mechanics [2,19].

\section{Theory of mixture in cylinder array}

\subsection{Assumptions}

Volume of mixture $\Omega$ is a fixed open system whose boundary $\partial \Omega$ is not time-dependent. Volume (resp. area) fraction $\phi_{\alpha}=\frac{V_{\alpha}}{V}$ (resp. $\varepsilon_{\alpha}=\frac{S_{\alpha}}{S}$ ) of $\alpha$-phase within $\Omega$ is constant for each cell since corresponding cylinder displacement is bounded by $\partial \Omega$. Therefore in each cell physical quantities associated with each phase may be defined as follows :

$$
\begin{aligned}
\rho_{\alpha} & =\frac{1}{V_{\alpha}} \int_{\Omega_{\alpha}} \rho(\mathbf{x}, t) d v, \\
\mathbf{v}_{\alpha}(t) & =\frac{1}{V_{\alpha}} \int_{\Omega_{\alpha}} \mathbf{v}(\mathbf{x}, t) d v \quad \text { and } \quad \mathbf{v}_{m}(t)=\frac{1}{V} \int_{\Omega} \mathbf{v}(\mathbf{x}, t) d v \\
& \Longrightarrow \quad \mathbf{v}_{m}(t)=\phi_{f} \mathbf{v}_{f}(t)+\phi_{s} \mathbf{v}_{s}(t) .
\end{aligned}
$$

Similarly time-related stress vectors of $\alpha$-phase at macroscopic scale are defined as :

$$
\begin{aligned}
\mathbf{T}_{\alpha}(t) & =\frac{1}{S} \int_{\partial \Omega_{\alpha}} \mathbf{T}(\mathbf{x}, t) d s \quad \text { and } \quad \mathbf{T}_{m}(t)=\frac{1}{S} \int_{\partial \Omega} \mathbf{T}(\mathbf{x}, t) d s \\
& \Longrightarrow \quad \mathbf{T}_{m}(t)=\varepsilon_{f} \mathbf{T}_{f}(t)+\varepsilon_{S} \mathbf{T}_{s}(t) .
\end{aligned}
$$

In what follows, mass conservation and momentum balance are formulated for a given volume of fluid solid mixture and lead to a set of constitutive equations for the singular behaviour of each fluid and solid phases [5,14,25,27-29].

\subsection{Conservation equations}

\subsubsection{Mass conservation}

For an open system change of mass $m$ in the mixture is given by :

$\frac{d m}{d t}=\frac{d}{d t} \int_{\Omega} \rho(\mathbf{x}, t) d v=-\int_{\partial \Omega} \rho(\mathbf{x}, t)\left(\mathbf{v}_{m}(t) \cdot \mathbf{n}\right) d s$

$\mathbf{n}$ is the unit outward vector normal to surface $\partial \Omega$. As the control volume is fixed, total time-derivative and partial-time derivative are equivalent, the divergence theorem may be applied and integration term may be split into two parts over both subdomains. Since time-derivatives of $\rho_{f}$ and $\rho_{s}$ are zero, this yields:

$$
\frac{\partial}{\partial t} \int_{\Omega_{f}} \rho_{f} d v+\frac{\partial}{\partial t} \int_{\Omega_{s}} \rho_{s} d v=-\int_{\Omega} \nabla \cdot\left(\rho(\mathbf{x}, t) \mathbf{v}_{m}(t)\right) d v=0 .
$$

The mixture mean velocity $\mathbf{v}_{m}$ is constant over $\Omega$ and it only depends on time. One finally gets the following mass conservation equation :

$$
\begin{aligned}
& \left(\rho_{f} \int_{\Omega_{f}} d v+\rho_{s} \int_{\Omega_{s}} d v\right) \nabla \cdot \mathbf{v}_{m}(t)=0 \\
& \Longrightarrow \quad \nabla \cdot\left(\phi_{f} \mathbf{v}_{f}(t)\right)+\nabla \cdot\left(\phi_{s} \mathbf{v}_{s}(t)\right)=0
\end{aligned}
$$

Eq. (1) is the mass conservation equation of the model formulated at the macroscopic scale to be considered.

\subsubsection{Momentum balance}

For an open system change of momentum quantity $q$ in the mixture may be given by :

$$
\begin{aligned}
\frac{d q}{d t} & =\frac{d}{d t} \int_{\Omega} \rho(\mathbf{x}, t) \mathbf{v}(\mathbf{x}, t) d v \\
& =\int_{\partial \Omega}\left[\mathbf{T}(\mathbf{x}, t)-\rho(\mathbf{x}, t) \mathbf{v}(\mathbf{x}, t)\left(\mathbf{v}_{m}(t) \cdot \mathbf{n}\right)\right] d s+\int_{\Omega} \mathbf{b}(\mathbf{x}, t) d v
\end{aligned}
$$

where $\mathbf{T}(\mathbf{x}, t)$ is the stress vector per surface unit, $\mathbf{b}(\mathbf{x}, t)$ the volumetric force vector per volume unit and $\mathbf{n}$ the unit outward vector normal to surface $\partial \Omega$. The divergence theorem may be applied and the integration over $\Omega$ may be split into two terms over $\Omega_{f}$ and $\Omega_{s}$ respectively as follows :

$$
\begin{aligned}
\frac{\partial}{\partial t} \int_{\Omega_{f}} \rho_{f} \mathbf{v}(\mathbf{x}, t) d v+\frac{\partial}{\partial t} \int_{\Omega_{s}} \rho_{s} \mathbf{v}(\mathbf{x}, t) d v \\
=-\int_{\Omega} \nabla \cdot\left(\rho(\mathbf{x}, t) \mathbf{v}(\mathbf{x}, t) \otimes \mathbf{v}_{m}(t)\right) d v \\
\quad+\int_{\partial \Omega} \mathbf{T}(\mathbf{x}, t) d s+\int_{\Omega} \mathbf{b}(\mathbf{x}, t) d v
\end{aligned}
$$

Density quantities $\rho_{\alpha}$ are homogeneously defined over $\Omega_{\alpha}$. This brings out the averaged velocity fields $\mathbf{v}_{\alpha}(t)$ and then, after dividing by $V=\int_{\Omega} d v$, brings also out its associated volume fraction $\phi_{\alpha}$. One finally gets the following momentum equation :

$$
\begin{aligned}
\frac{\partial}{\partial t}[ & \left.\phi_{f} \rho_{f} \mathbf{v}_{f}(t)\right]+\frac{\partial}{\partial t}\left[\phi_{s} \rho_{s} \mathbf{v}_{s}(t)\right] \\
= & -\nabla \cdot\left(\phi_{f} \rho_{f} \mathbf{v}_{f}(t) \otimes \mathbf{v}_{m}(t)\right)-\nabla \cdot\left(\phi_{s} \rho_{s} \mathbf{v}_{s}(t) \otimes \mathbf{v}_{m}(t)\right) \\
& +\frac{1}{V} \int_{\partial \Omega} \mathbf{T}(\mathbf{x}, t) d s+\frac{1}{V} \int_{\Omega} \mathbf{b}(\mathbf{x}, t) d v
\end{aligned}
$$

Eq. (2) represents the momentum balance equation of the model formulated at the macroscopic scale to be considered.

\subsection{Closure problem}

\subsubsection{Stress vector}

According to previous assumptions, one can write :

$\int_{\partial \Omega} \mathbf{T}(\mathbf{x}, t) d s=\int_{\partial \Omega} \mathbf{T}_{m}(t) d s=\int_{\partial \Omega}\left(\varepsilon_{f} \mathbf{T}_{f}(t)+\varepsilon_{s} \mathbf{T}_{s}(t)\right) d s$ 


$$
=\int_{\partial \Omega} \mathbf{T}_{f}(t) d s
$$

The fluid stress vector is related to mean hydrostatic pressure $p_{f}(t)$ and mean deviatoric tensor $\tau_{f}(t)$, both over $\Omega_{f}$. The flow is assumed to be incompressible and the viscosity $\mu_{f}$ to be homogeneous over $\Omega_{f}$. Therefore, in the context of classical newtonian fluid dynamics formulation, the stress vector satisfies :

$$
\begin{aligned}
\int_{\partial \Omega} \mathbf{T}(\mathbf{x}, t) d s & =\int_{\partial \Omega}\left[-p_{f}(t) I+\tau_{f}(t)\right] \cdot \mathbf{n} d s \\
& =\int_{\partial \Omega}\left[-p_{f}(t) I+\mu_{f}\left(\nabla \mathbf{v}_{f}(t)+{ }^{t} \nabla \mathbf{v}_{f}(t)\right)\right] \cdot \mathbf{n} d s
\end{aligned}
$$

where $I$ is the unit tensor. The divergence theorem combined with the homogeneous features of both averaged pressure and fluid velocity quantities finally leads to :

$\int_{\partial \Omega} \mathbf{T}(\mathbf{x}, t) d s=\left[-\nabla p_{f}(t)+\nabla \cdot \mu_{f}\left(\nabla \mathbf{v}_{f}(t)+{ }^{t} \nabla \mathbf{v}_{f}(t)\right)\right] \int_{\Omega} d v$

\subsubsection{Volumetric force}

No volumetric force acts over the volume of fluid material. However the cylinder included into the mixture gives rise to a resistance in response to the applied external load according to its oscillating behaviour. Indeed, the cylinder belonging to a given mixture $\Omega$ is assumed to be flexibly mounted. Its motion is governed by the following dynamics equation :

$M \frac{\partial^{2} \mathbf{u}_{s}}{\partial t^{2}}(t)+K \mathbf{u}_{s}(t)=\mathbf{F}(t)$,

where $\mathbf{u}_{s}(t)$ designates the cylinder displacement from its equilibrium position within $\Omega$ and $\mathbf{F}(t)$ the fluid load arising on outer cylinder interface. $M$ and $K$ are the mass and effective stiffness of the cylinder respectively. The resistance level is characterized by the effective stiffness $K$ whose nature is volumetric and which is assumed to be homogeneous over $\Omega_{s}$. Therefore, the volumetric force of a given fluid-cylinder mixture $\Omega$ satisfies :

$\int_{\Omega} \mathbf{b}(\mathbf{x}, t) d v=\int_{\Omega}-\frac{K}{V} \mathbf{u}_{s}(t) d v=-\frac{K}{V} \mathbf{u}_{s}(t) \int_{\Omega} d v$

\subsubsection{Resulting momentum equation}

According to previous derivations the resulting momentum equation within a given volume of fluid-cylinder mixture is such that :

$$
\begin{aligned}
\frac{\partial}{\partial t}\left[\phi_{f} \rho_{f} \mathbf{v}_{f}(t)\right]= & -\nabla \cdot\left(\phi_{f} \rho_{f} \mathbf{v}_{f}(t) \otimes \phi_{f} \mathbf{v}_{f}(t)\right)-\nabla \cdot\left(\phi_{s} \rho_{s} \mathbf{v}_{s}(t) \otimes \phi_{f} \mathbf{v}_{f}(t)\right) \\
& -\nabla \cdot\left(\phi_{f} \rho_{f} \mathbf{v}_{f}(t) \otimes \phi_{s} \mathbf{v}_{s}(t)\right)-\nabla \cdot\left(\phi_{s} \rho_{s} \mathbf{v}_{s}(t) \otimes \phi_{s} \mathbf{v}_{s}(t)\right) \\
& -\nabla p_{f}(t)+\nabla \cdot \mu_{f}\left(\nabla \mathbf{v}_{f}(t)+{ }^{t} \nabla \mathbf{v}_{f}(t)\right)-\frac{1}{V} \mathbf{F}(t)
\end{aligned}
$$

The set of Eqs. (1), (3) and (4) constitutes the macroscopic model. It displays 8 scalar unknowns and 5 scalar equations in a two-dimensional configuration. Therefore this system needs to be closed.

In what follows, one introduces a hydrodynamic load formulation involving others unknowns, namely averaged fluid and solid velocity fields.

\section{Hydrodynamic load model}

\subsection{Load model formulation}

The dynamical response of a beam under fluid flow may be significantly affected by surrounding viscous fluid. Therefore accurate estimates for near-wall fluid load and associated flow perturbation are required for prediction of dynamical response, especially in presence of large size cylinder arrangement. Moreover a consistent model of hydrodynamic effects must be suitable for vibrating cylinders as well as for fixed ones, i.e. in a dynamic and in a static contexts. Indeed, a robust estimate of fluid load exerted on a fixed cylinder (friction effects) anywhere in the large array, is required to enable a good flow prediction elsewhere in the array made up of both vibrating and fixed cylinders. [21] or [15] models should be adapted since formulations they are relying on are linear with the structure velocity and acceleration, and thus only concern moving cylinders.

In [12] one introduces an extension of Morison's equation [13] to predict hydrodynamic effects acting on moving offshore slender structures, by adding to this equation two additional terms depending on the moving solid variables. Inspired by this approach, one replaces the propagation wave velocity by the averaged flow velocity surrounding the cylinder :

$$
\begin{aligned}
\frac{\mathbf{F}(t)}{\Delta z}= & \rho_{f}\left(1+C_{m f}\right) \frac{\pi D^{2}}{4} \frac{\partial \mathbf{v}_{f}(t)}{\partial t}-\rho_{f} C_{m s} \frac{\pi D^{2}}{4} \frac{\partial \mathbf{v}_{s}(t)}{\partial t} \\
& +\frac{1}{2} \rho_{f} D V^{*}\left(C_{d f} \mathbf{v}_{f}(t)-C_{d s} \mathbf{v}_{s}(t)\right)+\frac{1}{2} \rho_{f} D C_{d 0} V_{\infty}^{2} \mathbf{n}_{f}
\end{aligned}
$$

where $\Delta z$ is the length cylinder, $D$ the diameter, $f_{s}$ the oscillation frequency in vacuum, $V_{\infty}$ the inlet cross flow velocity, $\mathbf{n}_{f}$ the unit vector following the inlet cross flow direction, $V^{*}=\max \left\{f_{s} D ; V_{\infty}\right\}$ the characteristic velocity of the system, $C_{m f}$ the inertia coefficient for fluid oscillation, $C_{s f}$ the inertia coefficient for cylinder oscillation, $C_{d f}$ the drag coefficient for fluid oscillation, $C_{s f}$ the drag coefficient for cylinder oscillation and $C_{d 0}$ the drag coefficient for steady cross flow. Eq. (5) involves 5 coefficients $C_{m f}, C_{d f}, C_{m s}, C_{d s}$ and $C_{d 0}$ which are unknown and must be evaluated for closure of the hydrodynamic load model. In what follows, one proposes a numerical closure method for evaluating these coefficients.

\subsection{Numerical closure method}

The present work involves a multi-physics multi-scale approach. The computational procedure to be proposed for evaluating the closure coefficients relies on numerical simulations performed at the microscopic scale on a reduced size domain involving a small number of moving and non-moving cylinders. Therefore the full computational procedure involves a coupling between macroscopic and microscopic computations performed in static and dynamic contexts.

\section{Step 1 : Microscopic-scale computation}

One defines a reduced-size domain assumed to be representative of fluid structure interaction occurring within a large cylinder array. This domain includes at least one non-moving cylinder and one moving cylinder. The numerical simulation to be performed on this domain at microscopic-scale provides flow fields in the vicinity of both cylinders.

A specific attention is paid to near-wall mesh refinement as depicted by Fig. 2. Initial and boundary conditions must be representative of those encountered in large-size arrangement for macroscopic scale simulation. Periodic boundary conditions are used in presence of an initial still fluid. In this way, microscopic-scale simulation provides accurate estimate of space distribution and time evolution of fluid load acting on both fixed and moving cylinders, as well as space-averaged flow fields and cylinder kinematics over each macroscopic cell.

In the present work these microscale solutions are used on one hand for closure of the macroscopic model, on the other hand for its validation by performing comparisons between macroscopic and space-averaged microscopic fields.

\section{Step 2 : Closure coefficient evaluation}

In order to determine the set of 5 unknown coefficients, two multiple linear regressions by a least-square method of hydrody- 


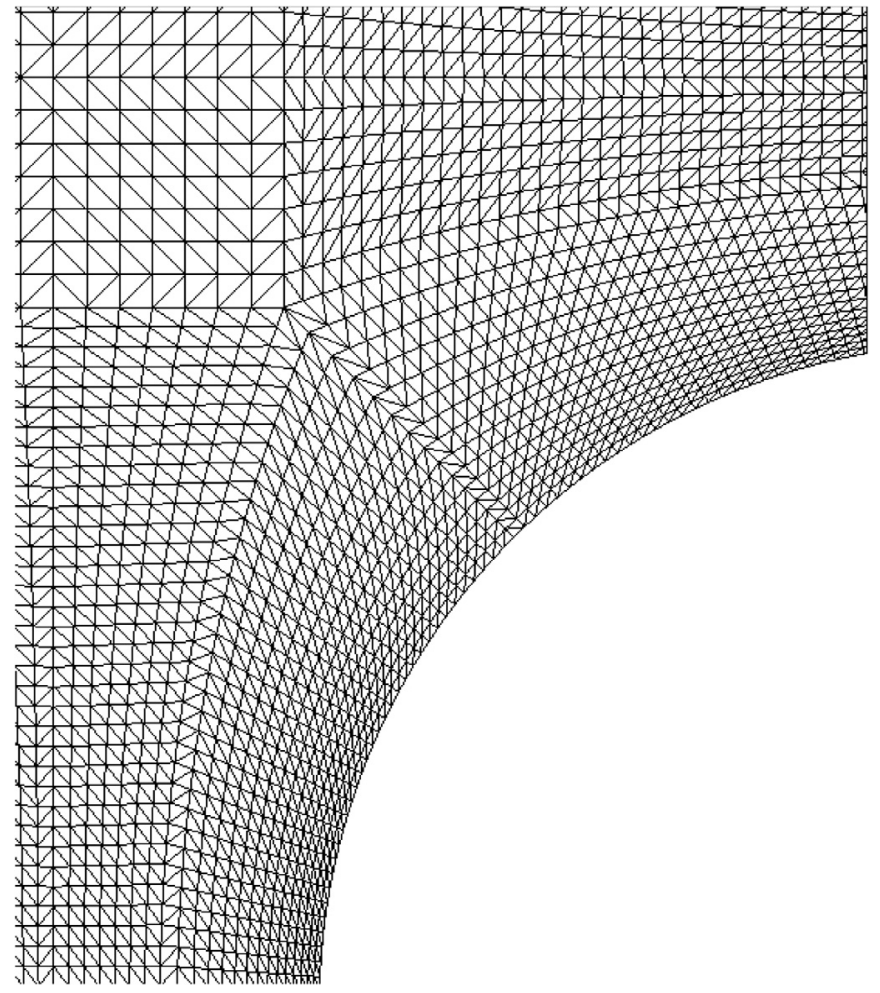

Fig. 2. Near-wall mesh refinement of some region of the computational domain involved for microscopic numerical simulation.
MicRoscopic SOLUTION

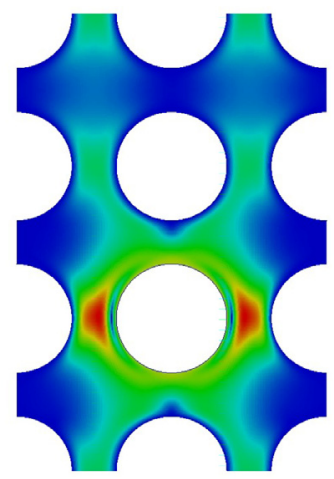

Averages over DISCRETE ELEMENTS

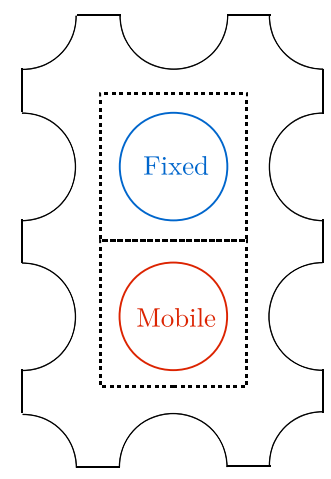

Fig. 3. Closure coefficient estimation procedure.

namic load evaluated at microscopic-scale are successively performed. From microscopic-scale solution, averaged fluid and solid velocities over each discrete element are computed. Then fluid contribution including drag and inertia coefficients, $C_{d 0}, C_{d f}$ and $C_{m f}$, are estimated from fluid load applied to the fixed cylinder. Next, $C_{d s}$ and $C_{m s}$ are estimated from fluid load on the moving cylinder. Finally one gets an evaluation of all closure coefficients to be introduced into the hydrodynamic load model.

In the present work the reduced domain involves one fixed cylinder and one moving cylinder embedded into a periodic cell simulating surrounding cylinders as depicted in Fig. 3. Depending on mechanical exchanges to be involved between fluid and solid in cylinder array, these 5 coefficients may be evaluated as
Table 1

Relative error on coefficients over the full-size domain.

\begin{tabular}{lllll}
\hline Coefficient & $C_{m f}$ & $C_{d f}$ & $C_{m s}$ & $C_{d s}$ \\
\hline Small-size domain & 1,46 & 4,70 & 1,41 & 5,13 \\
Full-size averaging & 1,65 & 5,08 & 1,42 & 4,88 \\
Relative error (\%) & 13,01 & 8,09 & 0,71 & 4,90 \\
\hline
\end{tabular}

a space tensor potentially time-dependent for possible bifurcation and transient modeling.

Step 3 : Correction step is needed

With the proposed method the reference reduced-scale domain required for coefficient estimation may be chosen differently depending on the problem. Therefore it is important to check the validity of coefficient distribution over the whole domain in order to introduce a correction if required. An example of coefficient space distribution over the whole domain is displayed in Fig. 4; cell-space averaged coefficients deduced from a microscopic computation over the whole domain are compared to estimates coming from a computation over the reduced-size domain.

Relative errors on coefficients $C_{m f}, C_{d f}$ in the external area and $C_{m s}, C_{d s}$ in the center part are provided by Table 1.

According to these results, if one excludes the interface area between static and dynamic parts, a relative homogeneous distribution of coefficients is ensured in the full domain. Therefore in the present configuration, the reduced-size domain is appropriate for coefficient computation. However in the presence of stronger dynamical effects, it may be required to rely on a larger and more representative domain.

\subsection{Full macroscopic model}

\subsubsection{Space discretization}

Mass conservation and momentum balance are established for a given volume of fluid solid mixture referred to as a discrete element of the whole cylinder arrangement. The mechanical exchange between solids and surrounding flow is written in terms of spacediscrete equations. By this way, let us consider the cylinder array to be divided into $n_{\text {elt }}$ identical discrete elements $\Omega_{i}, \forall$ integer $i \in[1$; $\left.n_{\text {elt }}\right]$. In what follows, the set of equations of the full macroscopic model is formulated. Time indice $(t)$ is omitted for sake of clarity. Therefore the fully coupled system is provided by Eqs. (6) to (9).

- Mass conservation within $\Omega_{i}$

$$
\nabla \cdot\left(\phi_{f} \mathbf{v}_{f i}\right)=-\nabla \cdot\left(\phi_{s} \mathbf{v}_{s i}\right)
$$

- Momentum balance within $\Omega_{i}$

$$
\begin{aligned}
\frac{\partial}{\partial t}( & \left.\phi_{f} \rho_{f} \mathbf{v}_{f i}\right)-\nabla \cdot\left(\phi_{f} \rho_{f} \mathbf{v}_{f i} \otimes \phi_{f} \mathbf{v}_{f i}\right) \\
= & -\nabla p_{f i}+\nabla \cdot \mu_{f}\left(\nabla \mathbf{v}_{f i}+{ }^{t} \nabla \mathbf{v}_{f i}\right)-\frac{1}{V} \mathbf{F}_{i} \\
& -\nabla \cdot\left(\phi_{f} \rho_{f} \mathbf{v}_{f i} \otimes \phi_{s} \mathbf{v}_{s i}\right)-\nabla \cdot\left(\phi_{s} \rho_{s} \mathbf{v}_{s i} \otimes \phi_{s} \mathbf{v}_{s i}\right) \\
& -\nabla \cdot\left(\phi_{s} \rho_{s i} \mathbf{v}_{s i} \otimes \phi_{f} \mathbf{v}_{f i}\right)
\end{aligned}
$$

- Solid displacement within $\Omega_{i}$

$$
M \frac{\partial^{2} \mathbf{u}_{s i}}{\partial t^{2}}+K \mathbf{u}_{s i}=\mathbf{F}_{i}
$$

- Hydrodynamic load modeling

$$
\begin{aligned}
\frac{\mathbf{F}_{i}}{\Delta z}= & \rho_{f}\left(1+C_{m f}\right) \frac{\pi D^{2}}{4} \frac{\partial \mathbf{v}_{f i}}{\partial t}-\rho_{f} C_{m s} \frac{\pi D^{2}}{4} \frac{\partial \mathbf{v}_{s i}}{\partial t} \\
& +\frac{1}{2} \rho_{f} D V^{*}\left(C_{d f} \mathbf{v}_{f i}-C_{d s} \mathbf{v}_{s i}\right)+\frac{1}{2} \rho_{f} D C_{d 0} V_{\infty}^{2} \mathbf{n}_{f_{i}}
\end{aligned}
$$



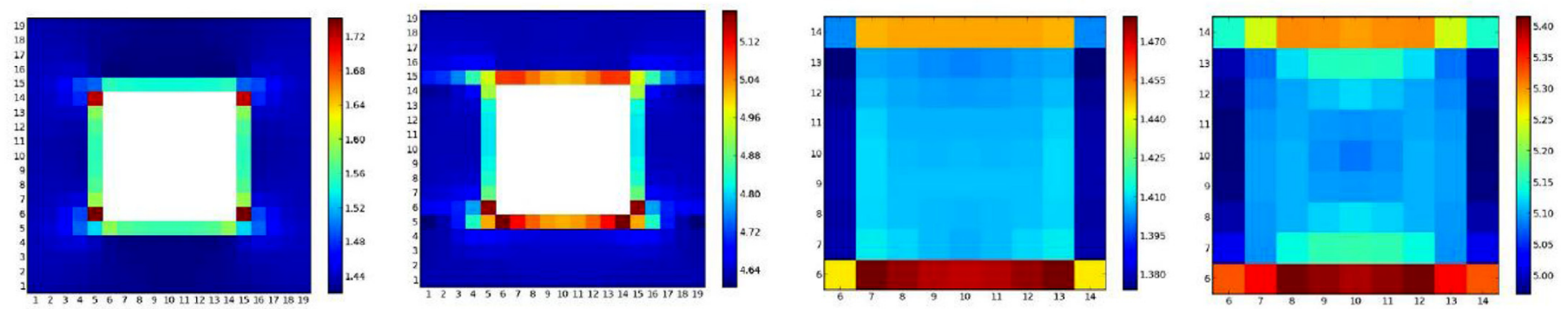

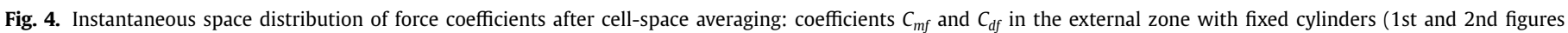
respectively) and coefficients $C_{m s}$ and $C_{d s}$ in the central region where cylinders are moving (3rd and 4th figures respectively).

\subsubsection{Time discretization}

Both macroscopic and microscopic scale computations are performed by using the same solver ${ }^{1}$ computing Navier-Stokes equations involving a cell-centered colocated finite volume method. In the present work time discretization is based on an implicit Euler scheme. Variables are supposed to be known at time $t^{n}$ and have to be computed at time $t^{n+1}$. At each time step $\Delta t=t^{n+1}-t^{n}$, velocity and pressure fields are computed in two steps:

- a prediction step in which the momentum equation is solved to compute the predicted velocity $\widetilde{\mathbf{v}}_{i}^{n+1}$ and pressure is taken explicit.

- a correction step in which $\widetilde{\mathbf{v}}_{i}^{n+1}$ is corrected to obtain $\mathbf{v}_{i}^{n+1}$ according to mass conservation equation.

All terms including a solid part contribution, mass and/or momentum source terms, are considered as explicit. Moreover microscopic scale computations rely on a moving grid method [1,10,11], provided that grid dynamics involved in microscopic simulation is limited by each macroscopic cell boundary for space-averaging purposes. Finally, the single-degree-of-freedom rigid solid dynamics equation is solved by using an unconditionaly stable Newmark scheme.

\section{Macroscopic numerical prediction of flow-induced vibration}

\subsection{Configuration}

The macroscopic model is evaluated on a configuration involving an array of 361 vibrating cylinders subjected to the same brief external load. Fluid and solid motions are considered in the crosssection plane of cylinders. The initial excitation drives cylinder oscillation only in the cross direction. The numerical solution provided by the macroscopic model is compared to the one given by the space-averaged microscopic-scale solution. Fig. 5 displays the system to be considered. At each time, the cylinder array is submitted to an inlet cross flow such that, in steady configuration, the interstitial flow Reynolds number and reduced velocity are respectively $R e=100$ and $V_{R}=0.91$. Numerical periodic conditions are assigned to both top and bottom boundaries to simulate a large computational domain, and also to prevent possible numerical pertubations related to wall boundary layer development. Table 2 summarizes material and geometrical parameters and Table 3 exhibits the set of estimated coefficients obtained according to the previously-mentioned computational procedures.

\subsection{Flow fields and solid response}

In each cell the macroscopic-scale model provides the time evolution of space-averaged flow velocity, flow pressure and, depending on the location within the array, cylinder displacement. These

\footnotetext{
${ }^{1}$ http://code-saturne.org/cms/.
}

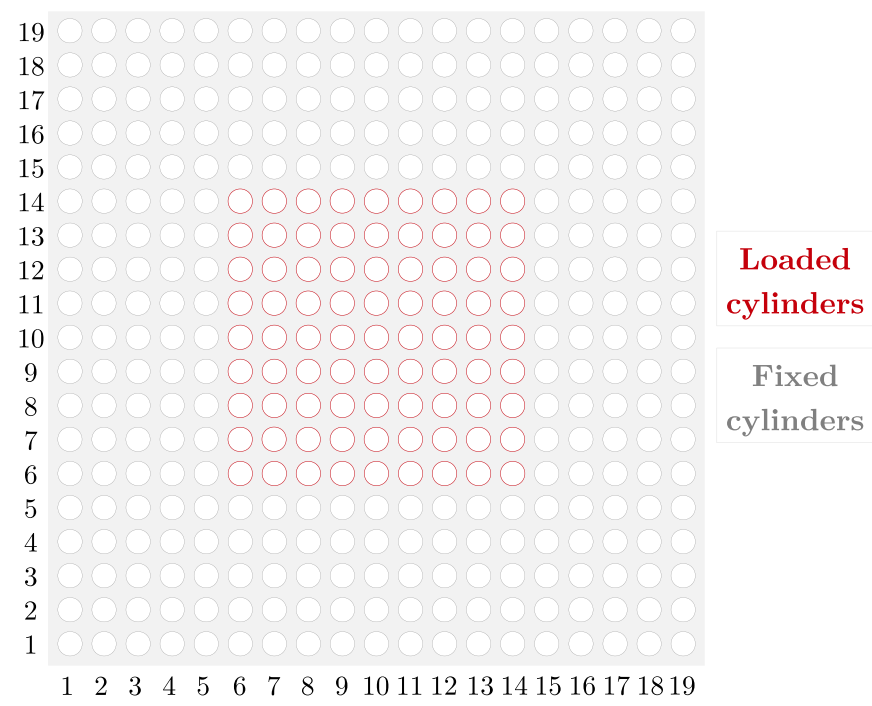

Fig. 5. Cylinder arrangement involving fixed (outside) and moving (inside) cylinders.

Table 2

System parameters.

\begin{tabular}{lll}
\hline Variable & Value & Unit \\
\hline$D$ & 10,0 & $\mathrm{~mm}$ \\
$P$ & 14,0 & $\mathrm{~mm}$ \\
$f$ & 1,1 & $\mathrm{~Hz}$ \\
$\rho_{s}$ & $3,0.10^{3}$ & $\mathrm{~kg} \cdot \mathrm{m}^{-3}$ \\
$\rho_{f}$ & $1,0.10^{3}$ & $\mathrm{~kg} \cdot \mathrm{m}^{-3}$ \\
$\mu_{f}$ & $1,0.10^{-3}$ & $\mathrm{~kg} \cdot \mathrm{m}^{-1} \cdot \mathrm{s}^{-1}$ \\
$V_{\infty}$ & $0,28 \cdot 10^{-2}$ & $\mathrm{~m} \cdot \mathrm{s}^{-1}$ \\
\hline
\end{tabular}

Table 3

Estimated coefficients

\begin{tabular}{lllll}
\hline $\mathrm{C}_{\mathrm{mf}}$ & $\mathrm{C}_{\mathrm{ms}}$ & $\mathrm{C}_{\mathrm{df}}$ & $\mathrm{C}_{\mathrm{ds}}$ & $\mathrm{C}_{\mathrm{d} 0}$ \\
\hline 1,67 & 1,47 & 5,34 & 4,94 & $-1,2$ \\
\hline
\end{tabular}

fields are compared to those obtained by averaging microscopicscale solutions over material volumes corresponding to these discrete elements. Table 4 shows instantaneous space distribution of averaged pressure and fluid velocity. The macro-scale solution reproduces with good agreement the space distribution of fields deduced from the micro-scale solution. In several macroscopic cells Table 5 shows time history of macro-scale solution compared to those obtained by micro-scale simulation. Figures focus on 4 elements, each one being associated with one moving cylinder. Each plot starts at the end of the initialization process. In each element the macroscopic-scale model reproduces with good agreement the 
Table 4

Comparison between averaged-microscopic (left) and macroscopic (right) solutions in terms of fluid velocity (top) and pressure (bottom).

\section{AVERAGED-MICROSCOPIC}
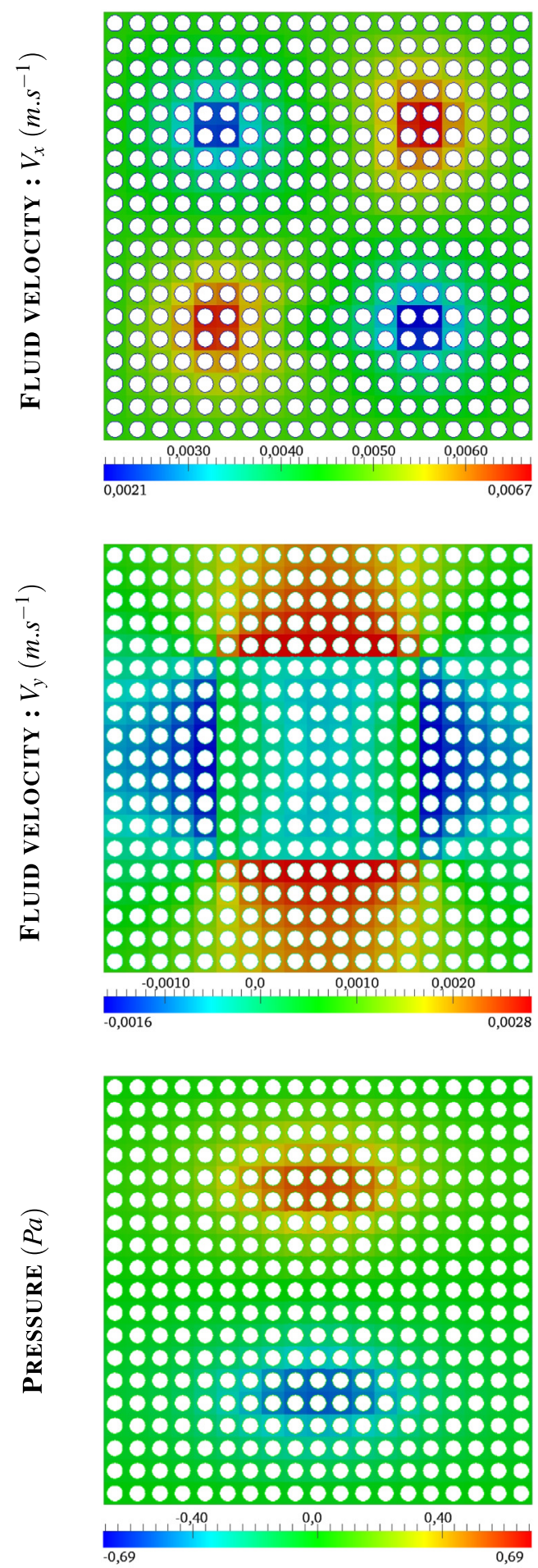

damping effect due to the surrounding viscous flow. No structural damping is included here. According to results the estimated error between microscopic and macroscopic solutions rises 3\%. For a same cell, the averaged fluid velocity after 6 periods may arise a phase delay but this does not significantly affect the cylinder displacement estimate.
MACROSCOPIC
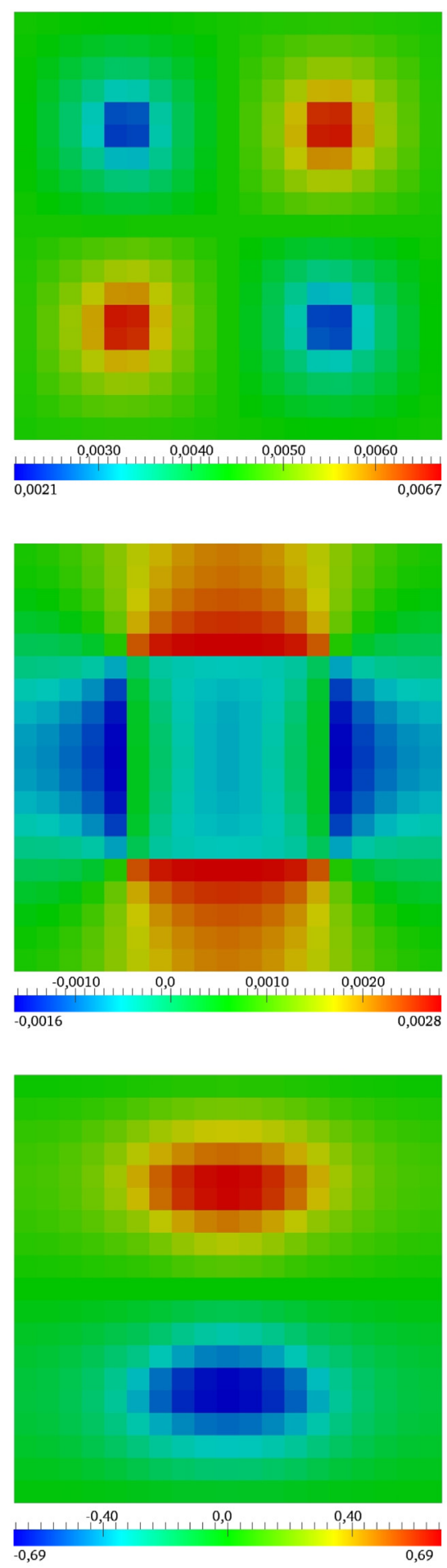

\subsection{Computing performances}

For the calculations involved in the present article, the microscopic-scale computation over the large array on 144 processors requires a computational time of 8 hours for 20 oscillation periods, whereas the macroscopic-scale computation on a single 
Table 5

Time evolutions of the macroscopic-scale solution, compared with those of the microscopic-scale solution for 4 discrete elements (from left to right) in terms of displacement (top), fluid velocity (middle) and pressure (bottom).
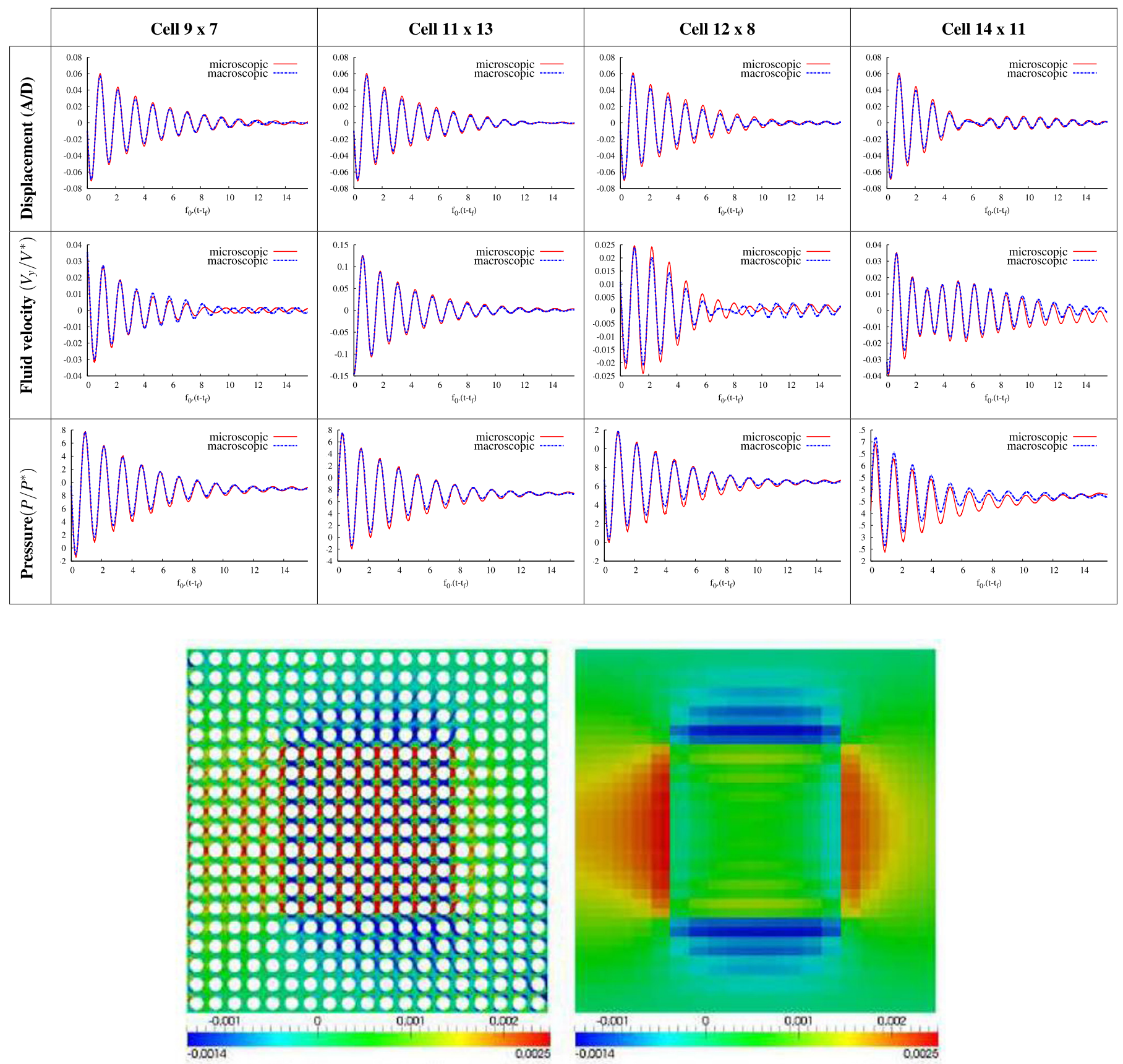

Fig. 6. Velocity field magnitude in the full domain computed through a microscopic (left) or a macroscopic (right) formulation.

processor requires a computational time of 50 minutes over the same period. Therefore the gain in CPU time provided by the proposed macroscopic model is evaluated to $99.9 \%$ provided that the reduced-size domain micro-scale computation CPU time required for the model closure may be neglected. One can find in Fig. 6 a comparison between instantaneous velocity field magnitudes computed at micro and macro scales in the full-size domain. According to these results, one can see that the macroscopic scale simulation is sufficient as far as one is interested in the energy transfer between the fluid and solids in order to account for a kind of solid effect distribution in the fluid domain.

\section{Concluding remarks}

Theory of mixture enables the derivation of a set of coupled equations describing fluid solid interaction in a large array of cylinders [16-18,26,30]. The formulated macroscopic model is built without any assumption on physical phenomena to be involved. In the present article the formulation is proposed in a twodimensional small magnitude motion context. Each macroscopic cell is referred to as a mixture including one cylinder with its surrounding fluid flow. Mechanical exchanges in fluid in solid and between fluid and solid are described both in a static and in a 
dynamic frameworks. The resulting set of equations involve fluid load acting on wall cylinders to be incorporated into the macroscopic model for closure. The closure consists in the evaluation of a set of 5 coefficients by using micro-scale reduced-size domain numerical simulations. This way the model developed and validated in the present work combines multi-physics (fluid-solid) and multi-scale (macro-microscopic) approaches. The validation is proposed on a configuration involving an array made up of 361 cylinders. The reference solution is the numerical one provided by a fully microscopic-scale computation performed on the same array. The macroscopic model reproduces the time history of the space-averaged solution with good agreement. The hydrodynamic load formulation involved in the model for closure seems to be appropriate for such cylinder array configurations. Further developments are now investigated in order to extend this formulation to three-dimensional configurations involving large magnitude displacements by using moving grid formulations.

\section{Declaration of Competing Interest}

The authors declare that they have no known competing financial interests or personal relationships that could have appeared to influence the work reported in this paper.

\section{References}

[1] Archambeau F, Méchitoua N, Sakiz M. A finite volume code for the computation of turbulent incompressible flows, industrial applications. Int J Finite Vol 2004;1.

[2] Atkin RJ, Craine RE. Continuum theories of mixtures:basic theory and historical developments. Q J Mech Appl Math 1976;29(209).

[3] Bedford A, Drumheller DS. Recent advances: theories of immiscible and structured mixtures. Int J Engng Sci 1983;21(8):863-960.

[4] De Groot S, Mazur. Non-equilibrium thermodynamics. Dover Books on Physics. Dover Publications; 1984

[5] Howes F, Whitaker S. The spatial averaging theorem revisited. Chem Eng Sci $1985 ; 40(8): 1387-92$.

[6] Jaquelin E, Brochard D, Trollat C, Jezequel L. Homogeneisation of non lineai arrau of confined beams. Nucl Eng Des 1996;165(1-2):213-23.

[7] Jus Y, Longatte E, Chassaing JC, Sagaut P. Low mass damping vortex induced vibrations of a single cylinder at moderate reynolds number. Journal of Pressure Vessel Technology 2014;136. 051305-1

[8] Khalak A, Williamson C. Dynamics of a hydroelastic cylinder with very low mass and damping. J Fluids Struct 1996;10(5):455-72.

[9] Lemos MD. Turbulence in porous media: modeling and application. Elsevier; 2006.
[10] Longatte E, Verraman V, Souli M. Time marching for simulation of fluid structure interaction problems. J Fluids Struct 2009;25(1):95-111.

[11] Longatte E, Baj F, Braza M, Hoarau Y, Ruiz D Canteneur C. Advanced numerica methods for uncertainty reduction on prediction of heat exchanger dynamic stability limits : review and perspectives. Nucl Eng Des 2013;258:164-75.

[12] Moe G, Verley RLP. An investigation into the hydrodynamic damping of cylinders oscillated in steady currents of various velocities. Report of the River and Harbour Laboratory, Norwegian Institute of Technology; 1978.

[13] Morison JR, O'Brien MP, Johnson JW, Schaff SA. The force exerted by surface waves on piles. In: Petroleum Trasactions, American Institute of Mining Engineers, vol. 189; 1950. p. 149-54

[14] Pekar M, Samohyl I. The thermodynamics of linear fluids and fluid mixtures Springer; 2014.

[15] Price SJ, Païdoussis MP. An improved mathematical model for the stability of cylinders rows subject to cross-flow. J Sound Vib 1984;97:615-40.

[16] Price SJ, Païdoussis MP. A single flexible cylinder analysis for the fluidelastic instability of an array of flexible cylinders in cross flow. ASME J Fluids Eng 1986;108:193-9.

[17] Price SJ, Païdoussis MP, Macdonald R, Mark B. THE flow induced vibration of a single flexible cylinder in a rotated suare array of rigid cylinders with pitch to diameter ratio of 212. J Fluids Struct 1987:1:359-78.

[18] Quintard M, Whitaker S. Transport in ordered and disordered porous media i: cellular average and the use of weighting functions. Transp Porous Media 1994:14:163-77.

[19] Quintard M, Whitaker S. Transport in ordered and disordered porous media ii: generalized volume arevaring. Transp Porous Media 1994;14:179-206.

[20] Ricciardi G, Bellizzi S, Collard B, Cochelin B. Modelling pressurized water reacteur cores in terms of porous media. J Fluids Struct 2009;25:112-13.

[21] Rogers RJ, Taylor C, Pettigrew MJ. Fluid effects on multi-span heat-exchanger tube vibration. In: ASME Pressure Vessel and Piping Conference, San Antonio, Texas; 1984.

[22] Sagaut P. Large Eddy Simulation for Incompressible Flows - An Introduction. Springer; 2005. Third Edition

[23] Shinde V, Marcel T, Hoarau Y, Deloze T, Harran G, Baj F, et al. Numerical simulation of the fluid structure interaction in a tube array under cross flow at moderate and high Reynolds number. J Fluids Struct 2014;47:99-113.

[24] Sigrist JF, Broc D. Dynamic analysis of a tube bundle with fluid-structure interaction modelling using a homogenisation method. Comput Methods Appl Mech Eng 2008;197:1080-99.

[25] Slattery J. Flow of viscoelastic fluids through porous media. A IChE] 1967;13(3):1066-71.

[26] Tanaka H, Tanaka K, Shimizu F. Fluidelastic analysis of tube bundle vibration in cross-flow. J Fluids Struct 2002;16(1):93-112.

[27] Veverka V. Theorem for the local volume average of a grandient revisited. Chem Eng Sci 1981;36:833-8.

[28] Whitaker S. Diffusion and dispersion in porous media. AIChE J $1967 ; 13(3): 420-7$.

[29] Whitaker S. Advances in theory of fluid motion in porous media. Ind Eng Chem 1969;61:14-28.

[30] Williamson C. Vortex dynamics in the cylinder wake. Annu Rev Fluid Mech 1996;28:477-539. 